\title{
TRIM16 Positively Regulates Osteogenic Differentiation of Human Periodontal Ligament Stem Cells by Modulating the Ubiquitination and Degradation of RUNX2
}

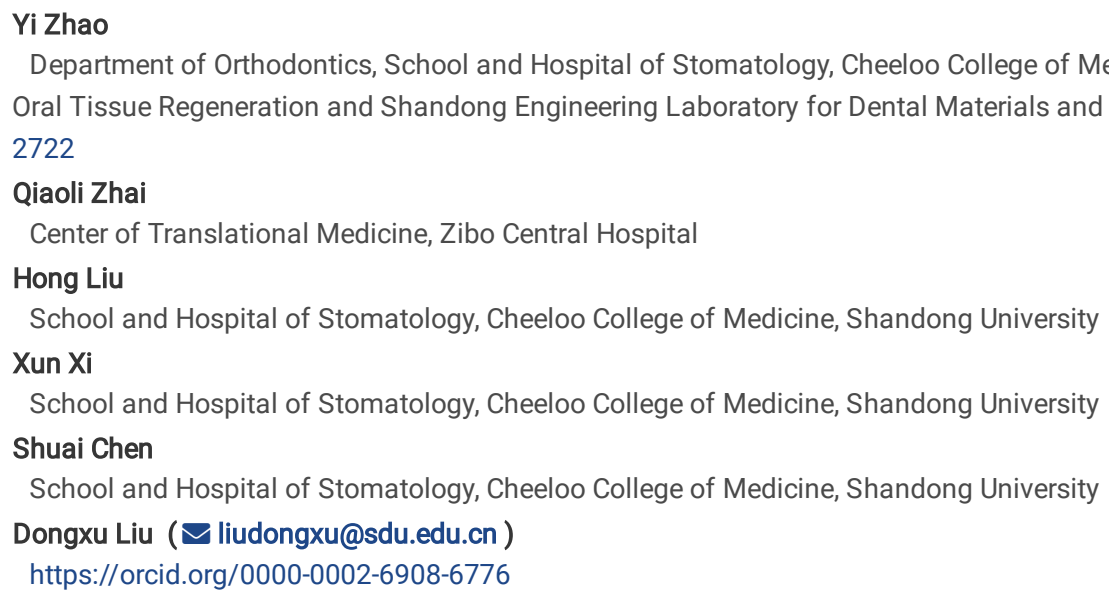




\section{Abstract}

\section{Background}

Periodontal disease is a common disease that compromises the integrity of tooth-supporting tissues. Bone regeneration is the ultimate goal of periodontal therapies, in which osteogenic differentiation of human periodontal ligament stem cells plays a critical role. The tripartite motif (TRIM) 16 is downregulated in periodontal tissues of patients with periodontitis and involved in osteogenic differentiation of human bone marrow mesenchymal stem cells(hBMSCs). However, the role of TRIM16 in the osteogenic differentiation of human periodontal ligament stem cells (hPDLSCs) is largely unknown.

\section{Methods}

hPDLSCs were isolated and identified by immunophenotype assays using flow cytometry. Overexpression plasmids and specific short-hairpin RNAs (shRNAs) were constructed to manipulate the expression of target molecules. Alkaline phosphatase (ALP) staining, alizarin red staining (ARS) and enzyme-linked immunosorbent assays (ELISA) were used to evaluate osteogenic potential capacity. Reverse transcription quantitative PCR (RT-qPCR) and Western blot analysis were performed to determine the expression of osteogenic-related markers and activation of relevant signaling pathways. Co-immunoprecipitation assays were performed to confirm the interactions between proteins and the ubiquitination of RUNX2. A LC-MS/MS analysis was performed to explore the different expression proteins in present of TRIM16.

\section{Results}

TRIM16 significantly promoted alkaline phosphatase activity and mineralized nodule formation, and positively regulated the osteogenic differentiation of hPDLSCs by enhancing protein expression of RUNX2, COL1A1 and OCN. Mechanistically, TRIM16 serves as a pivotal factor that stabilizes RUNX2 protein levels by decreasing CHIP-mediated K48-linked ubiquitination degradation of the RUNX2 protein. Besides, TRIM16 significantly increased expression of COL1A1 via activation of p38MAPK/RUNX2.

\section{Conclusion}

This study identified a novel mechanism of TRIM16 in regulating stability of the RUNX2 protein, which may promote the osteogenic differentiation of hPDLSCs. TRIM16 may be a potential target of stem cell based-bone regeneration for periodontal therapies.

\section{Introduction}

Periodontal diseases represent a broad range of progressive inflammatory conditions characterized by irreversible attachment loss and bone resorption[1]. Stem cell-based approaches coupled with scaffolding and guiding biomaterials have been used to promote bone regeneration, the ultimate goal of periodontal therapies[2]. Mesenchymal stem cells (MSCs) isolated from the human periodontal ligament (hPDLSCs) may differentiate into osteogenic, chondrogenic, adipogenic, and neurogenic cell lineages in vitro[3]. Because hPDLSCs have the ability to self-replenish and produce differentiated, multilineage progeny, these cells tend to regenerate their tissue of origin[4,5]. Periodontal ligament cells derived from autologous tissue have been implanted into periodontal lesions[6]; however, stem cell therapy still has many safety and regulatory barriers[1]. The tightly regulation of osteogenic differentiation of hPDLSCs is important for promoting tissue regeneration and maintaining dynamic homeostasis in the periodontaltissue.

Several signaling pathways have been implicated in the osteogenic differentiation of hPDLSCs, including Hedgehog, Fgf, Wnt, Pthlh and MAPK[7-9]. These pathways may play critical roles in regulating osteogenesis-related factors such as RUNX2, which is essential for bone formation because it induces the expression of major bone matrix proteins such as COL1A1, SPP1 and BGLAP2 in vitro[10, 11]. The activity and stability of RUNX2 are modulated at the posttranscription and post-translation levels [12-14]. Increasing evidence has shown that the ubiquitin-proteasome system is also involved in modulating osteogenesis[15, 16]. The ubiquitin-protein ligases (E3), the most abundant group of ubiquitination enzymes, have been shown to play an important role in bone formation by controlling protein levels of several receptor tyrosine kinases, signaling molecules and transcription factors. For example, Ubiquitin E3 ligase Itch negatively regulates osteoblast function by promoting proteasome degradation of osteogenic proteins[17]. The carboxy terminus of HSP70 interacting protein (CHIP)/STUB1 enhanced ubiquitination and degradation of SMAD proteins, and negatively regulated RUNX2 protein stability via a ubiquitination-dependent degradation[18].

TRIM16 belongs to the tripartite motif (TRIM) family of proteins, which include $~ 75$ proteins with E3 ligase activities and diverse functions in cell proliferation, differentiation, apoptosis, carcinogenesis and autophagy[19, 20]. TRIM16, devoid of a typical RING domain, harbors two B-box domains, a coiled-coil domain and a C-terminal domain. TRIM16 has been shown to possess auto-polyubiquitination activity and to act as an E3 ubiquitin ligase in vivo and in vitro[20]. TRIM16 is ubiquitously expressed, and is localized predominantly in the cytosol, while it may be translocated to the nucleus in the G1 phase of the cell cycle to affect cell cycle progression and cellular differentiation in neuroblastoma[21]. TRIM16 is also known as a tumor suppressor by inhibiting the epithelial-tomesenchymal transition (EMT) in non-small cell lung cancer and prostate cancer cells[22, 23]. Additionally, TRIM16 has been shown to interact with other proteins such as NRF2, p62 or galectin-3 by providing a platform for the assembly of core autophagic machineryin response to oxidative or proteotoxic stress[24, 25].

Previous studies have defined the functions of TRIM16 in several biological processes; Recently, significant downregulation of TRIM16 was observed in periodontal tissue of patients with periodontitis compared with tissues in healthy individuals[26]. We speculated the impaired osteogenic capacity of hPDLSCsin inflammatory microenvironment might be associated with lower expression of TRIM16. Thus, the biological roles of TRIM16 in osteogenic differentiation of hPDLSCsrequires further investigation. 
In this study, we investigated the functions of TRIM16 during the osteogenic differentiation of hPDLSCs. We found that TRIM16 promoted the osteogenic differentiation of hPDLSCsby decreasing CHIP-mediated degradation of RUNX2 and upregulating the expression of COL1A1 by activation of p38MAPK. Therefore, targeting TRIM16 may represent a potential therapeutic strategy to promote bone regeneration in patients with periodontal disease.

\section{Methods}

\subsection{Cell culture}

This study was approved by the Medical Ethics Committee of the School of Stomatology, Shandong University. hPDLSCs were obtained from premolars of healthy teenagers (aged 14-20) extracted for orthodontic purposes. Tissues from the middle third of the tooth root were collected, minced into small fragments of approximately $1-2 \mathrm{~mm}^{3}$, then digested in a solution of $3 \mathrm{mg} / \mathrm{ml}$ collagenase type I (Sigma-Aldrich, St. Louis, MO, USA) and $4 \mathrm{mg} / \mathrm{ml}$ dispase (Sigma-Aldrich) at $37^{\circ} \mathrm{C}$ for $1 \mathrm{~h}$. The primary cells were cultured with a-MEM (Biological Industries, Beit Haemek, Israel) containing 20\% fetal bovine serum (FBS) (Biological Industries) at $37^{\circ} \mathrm{C}$ with $5 \%$ carbon dioxide. The medium was replaced with fresh medium every three days until the cell monolayer reached $90 \%$ confluence. Cells were then passaged at a dilution ratio of 1:2 to expand the culture in 10\% FBS medium. Cells from passages 3-7 were used for subsequent experiments.

The stemness of hPDLSCs was characterized by phenotype analysis of hPDLSCs. Cells were trypsinized and washed with PBS and then incubated with CD90FITC, CD44-PE and PE-negative cocktail (CD45-PE, HLA-DR-PE). After incubation at $4^{\circ} \mathrm{C}$ protected from light for $1 \mathrm{~h}$, the cells were washed with PBS and flow cytometry was performed with a BD Accuri ${ }^{\mathrm{TM}} \mathrm{C} 6$ flow cytometer (BD Biosciences, San Jose, CA, USA).

The HEK293T cell line was obtained from the American Tissue Culture Collection (ATCC) and was cultured in DMEM (Sigma-Aldrich, St Louis, MO, USA) with $10 \% \mathrm{FBS}$ and $100 \mu \mathrm{g} / \mathrm{ml}$ penicillin-streptoMycin sulfate (Sigma-Aldrich) in a $5 \% \mathrm{CO} 2$ humidified atmosphere at $37^{\circ} \mathrm{C}$.

\subsection{In vitro differentiation assays}

Osteogenic induced medium contained complete culture medium supplemented with $100 \mathrm{nM}$ dexamethasone (Solarbio, Beijing, China), $10 \mathrm{mM} \beta$ glycerophosphate (Solarbio) and $50 \mathrm{mg} / \mathrm{l}$ ascorbic acid (Solarbio). Adipogenic medium contained complete culture medium supplemented with $1 \mu \mathrm{M}$ dexamethasone (Solarbio), $0.2 \mathrm{mM}$ indomethacin (Solarbio), $0.01 \mathrm{~g} / \mathrm{l}$ insulin (Solarbio) and 0.5mM isobutyl-methylxanthine (Solarbio). Cells were cultured in 24-well plates with osteogenic differentiation medium or adipogenic differentiation medium. The medium was replaced every other day. Osteogenic differentiation was evaluated with Alkaline phosphatase (ALP) and Alizarin red (AR) staining (Sigma-Aldrich). Adipogenic differentiation was evaluated with Oil Red $\mathrm{O}$ staining (Solarbio). All methods followed protocols recommended by the manufacturer.

\subsection{Plasmid construction and transfection}

To stably express TRIM16, the full-length open reading frame (ORF) of TRIM16 (GenBank NM_001348120) was amplified from a Human Multiple Tissue cDNA Panel (BD Biosciences) and then cloned into pLVX-puro at the Xhol and EcoRI sites. To knock down TRIM16, siRNA targeting TRIM16 and a scramble sequence were synthesized by Invitrogen (Beijing, China). The sequence of shRNA against TRIM16 and a negative control were then inserted into the pLKO.1 vector and named sh-TRIM16 and sh-NC, respectively. The transcripts of CHIP (GenBank NM_005861) and RUNX2 (GenBank NM_001024630) were amplified by PCR and subcloned into the pCMV-3Tag6 (Agilent Technologies, Santa Clara, CA, USA) and pCMV-Myc (Clontech, Mountain View, CA, USA) vectors, respectively. The constructs were named Flag-CHIP or Myc-RUNX2. All constructs were confirmed by direct sequencing (Table 1). pRK5-HA-Ubiquitin-WT and K48, K63 were gifts from Ted Dawson (Addgene, Watertown, MA, USA; plasmid \# 17608; 17605, 17606).

\subsection{RNA isolation and RT-qPCR assays}

Total RNA was extracted from hPDLSCs using TRIzol® reagent (Thermo Fisher Scientific, Waltham, MA, USA) according to the manufacturer's protocol. Total RNA was quantified using a NanoDrop 2000 (Thermo Fisher) and 1000 ng of total RNA was reverse-transcribed using a PrimeScript ${ }^{\text {TM }}$ RT reagent kit (Takara Bio, Shiga, Japan). Gene expression levels were determined by RT-qPCR using an ABI 7500 Real-Time PCR System (Thermo Fisher). RT-qPCR was performed in a $10 \mu$ reaction volume with a TB Green PCR Core Kit (Takara Bio) according to the manufacturer's instructions. The expression of $\beta$-actin was used for normalization. Changes in gene expression were calculated by the $2^{-\Delta \Delta C t}$ method. Primer sequences are listed in Table 2.

\subsection{Western blot analysis and co-immunoprecipitation}

Protein lysates were extracted from hPDLSCs with RIPA buffer (Solarbio) supplemented with 1\% protease inhibitors (Boster Bio, Wuhan, China) and quantified by a BCA Protein Assay Kit (Thermo Fisher). Equal amounts of total protein for each sample were separated by electrophoresis at $120 \mathrm{~V}$ for 90 min on a $10 \%$ sodium dodecyl sulfate polyacrylamide (SDS-PAGE) gel, and then transferred to polyvinylidene fluoride (PVDF) membranes (Merck Millipore, Burlington, MA,

USA) by electroblotting at $200 \mathrm{~mA}$ for $2 \mathrm{~h}$. The membranes were blocked with $5 \%$ nonfat milk. The primary antibodies of target proteins were incubated at $4{ }^{\circ} \mathrm{C}$ overnight, and secondary antibodies were incubated at room temperature for $1 \mathrm{~h}$. Finally, protein expression levels were quantitated by measuring the relative intensities of the bands using ImageJ software 5.0 (National Institutes of Health, Bethesda, MA, USA).

For co-immunoprecipitation, whole-cell extracts were lysed in IP lysis buffer composed of $25 \mathrm{mM}$ Tris-HCl pH 7.4, $150 \mathrm{mM} \mathrm{NaCl,} 1 \mathrm{mM}$ EDTA, $1 \%$ NP-40 and $5 \%$ glycerol and a protease inhibitor 'cocktail' (Merck Millipore). Briefly, the supernatant was collected and incubated with protein $\mathrm{G}$-Agarose for $2 \mathrm{~h}$ to remove nonspecific binding. The lysate was mixed with antibodies and gently rotated at $4^{\circ} \mathrm{C}$ for $4 \mathrm{~h}$, then protein G-Agarose was added to the lysate for overnight incubation. The beads were washed five times with IP buffer. Co-precipitated proteins were eluted with SDS-loading buffer at $95^{\circ} \mathrm{C}$ for 5 min and then analyzed using Western blotting as previous described. 
HEK293T cells transiently transfected with the indicated plasmids were cultured for $24 \mathrm{~h}$, then collected using IP lysis buffer. Antibodies were added to the lysate and gently rotated at $4^{\circ} \mathrm{C}$ for $4 \mathrm{~h}$, then protein G-Agarose was added to the lysate at $4^{\circ} \mathrm{C}$. After overnight incubation, the beads were washed five times with IP buffer and the immunoprecipitated proteins were eluted with SDS-loading buffer at $95^{\circ} \mathrm{C}$ for 5 min, then analyzed with Western blotting.

\subsection{Enzyme-linked immunosorbent assay (ELISA)}

To further evaluate osteogenic capacity, levels of osteocalcin (OCN) were assessed by the Human Osteocalcin ELISA Kit (ab195214, Abcam, Cambridge, MA, USA) according to the manufacturer's instructions. The optical density values were read by a microplate reader at $450 \mathrm{~nm}$. Protein levels are calculated by subtracting values from the medium and standardizing them according to the number of cells.

\subsection{LC-MS/MS and data analysis}

hPDLSCs were transfected with a pLVX-TRIM16 or pLVX-puro lentiviral vector and collected after a 14-day incubation in osteogenic medium. A filter aided sample preparation (FASP) method was used to generate tryptic peptides from crude lysates for LC-MS/MS detection. After a series of optimization processing, the LC-MS/MS mass spectrometry data were compared with the database for similarity scoring and protein identification using ProteinPilot ${ }^{\mathrm{TM}}$ V4.5. Each protein containing at least one unique peptide segment was considered to be a credible protein, and differentially expressed proteins were defined using the following cut-off criteria: $[\log F C]>1$ and p-value $<0.05$.

\subsection{Reagents and antibodies}

MG132, cycloheximide, and 3-MA were obtained from Sigma-Aldrich (St. Louis, MO, USA); SB203580 was from Selleck Chemicals (Houston, TX, USA); antiMyc, anti-Flag, anti-hemagglutinin (HA), anti-GAPDH, protein $\mathrm{G}$ agarose used for IP and horseradish peroxidase-conjugated secondary antibodies were from Santa Cruz Biotechnology (Dallas, TX, USA); anti-RUNX2, anti-p-p38 were obtained from Cell Signaling Technology (Beverly, MA, USA); anti-TRIM16, anti-CHIP were from Proteintech (Rosemont, IL, USA); anti-COL1A1 was from Servicebio (Wuhan, China).

\subsection{Statistical analysis}

Each experiment was performed in triplicate. Data were presented as the means \pm standard deviations. Statistical analysis was performed by Student's t-test or one-way analysis of variance (ANOVA) with GraphPad software (GSL Biotech LLC, San Diego, CA, USA). P-values $<0.05$ were considered statistically significant.

\section{Results}

\subsection{TRIM16 expression in hPDLSCs was enhanced during osteogenic differentiation}

hPDLSCs derived from periodontal ligament explants had a long spindle-like morphology and were arranged in whirlpool formations (Fig. 1A). To determine the multi-differentiation capacity of these hPDLSCs, Alizarin Red-positive mineralized matrix (Fig. 1B) and Oil Red O-positive lipid droplets (Fig. 1C) were observed after osteogenic and adipogenic induction, respectively. The immunophenotype assay showed that MSC-specific surface markers (CD90, CD44) were positive, while the typical hematopoietic and endothelial cell-specific markers (CD45 and HLA-DR) were negative (Fig. 1D), indicating that the primary hPDLSCs exhibited mesenchymal stem cell characteristics.

In an effort to profile TRIM16 expression patterns during osteogenic differentiation of hPDLSCs, we performed RT-qPCR and Western blotting. The results showed that TRIM16 mRNA and protein expression were enhanced during the commitment to an osteogenic linage (Fig. 1E-G), suggesting that TRIM16 may play an important role in the osteogenic differentiation of hPDLSCs.

\subsection{TRIM16 promoted the osteogenic differentiation of hPDLSCs}

To investigate the potential functions of TRIM16 during osteogenic differentiation of hPDLSCs, a TRIM16-overexpressing lentiviral vector was constructed and transfected into hPDLSCs. Concurrently, a TRIM16 specific shRNA was used to knockdown its expression. Efficacy was determined by RT-qPCR and Western blotting (Fig. 2A and B). To evaluate the capacity of osteogenic differentiation, ALP staining and Alizarin red staining were performed after treating with osteogenic induction media for 7 days or 14 days. The results showed that overexpression of TRIM16 significantly increased ALP activity and mineralized nodule formation, which were inhibited by knockdown of TRIM16 (Fig. 2C and D). In an ELISA assay, levels of OCN were significantly higher in the presence of TRIM16 than in the control (Fig. 2E). In assessment of mRNA and protein levels of several osteogenic-related markers, TRIM16 increased mRNA expression of SP7 and COL1A1 (Fig. 2G and H) and upregulated protein expression of COL1A1 and RUNX2 (Fig. 2I). Knockdown of TRIM16 significantly reduced mRNA expression of SP7 and COL1A1 (Fig. 2G and H) and protein levels of COL1A1 and RUNX2 (Fig. 2I). Interestingly, RUNX2 mRNA levels were not upregulated by TRIM16 and were not downregulated by knockdown of TRIM16 (Fig. 2F), indicating that TRIM16 had no effects on RUNX2 mRNA expression, but significantly influenced RUNX2 protein expression.

\subsection{TRIM16 reduced ubiquitination degradation of RUNX2}

To determine the mechanism of TRIM16-mediated stability of RUNX2, we used HEK293T cells with various doses of TRIM16 overexpression plasmid to verify changes in RUNX2 protein levels (Fig. 3A). TRIM16 consistently increased RUNX2 protein expression in a dose-dependent manner. The TRIM family of proteins has been implicated in the positive regulation of several critical transcription factors by reducing their ubiquitin-proteasome degradation; therefore, we 
inferred that TRIM16 could inhibit protein degradation of RUNX2. HEK293T cells were treated with the protein biosynthesis inhibitor cycloheximide (CHX) and the proteasome inhibitor MG-132 for various amounts of time. To rule out whether TRIM16 inhibited RUNX2 degradation via autophagy, HEK293T cells were treated with different concentrations of autophagy inhibitor 3-MA. The results showed that TRIM16 retarded the reduction of RUNX2 protein compared to the control group (Fig. 3B). TRIM16-induced RUNX2 stability was enhanced by MG-132 (Fig. 3C) but was not affected by 3-MA (Fig. 3D), indicating that TRIM16 inhibited the proteasomal degradation of RUNX2. To determine whether TRIM16 affected RUNX2 ubiquitination, Myc-RUNX2 was co-transfected with HAubiquitin and TRIM16 or empty vector into HEK293T cells. Overexpression of TRIM16 reduced the ubiquitination of RUNX2 (Fig. 3E). Because K48-linked and k63-linked poly-ubiquitination of proteins are two principal delivery signals in the ubiquitination system, we performed immunoprecipitation assays in the presence of two variants of ubiquitin. These variants can only be ubiquitinated at the lysine 48 residue (HA-K48-UB) or at the lysine 63 residue (HA-K63-UB). Overexpression of TRIM16 reduced K48-linked poly-ubiquitination of RUNX2 but increased K63-linked poly-ubiquitination of RUNX2 (Fig. 3F). In addition, vectors expressing Myc-RUNX2 and TRIM16 were co-transfected into 293T cells in a co-immunoprecipitation assay to demonstrate that TRIM16 physically interacts with RUNX2(Fig. 3G), and the interaction between TRIM16 and RUNX2 was also observed in hPDLSCs(Fig. 3H).All the results showed that RUNX2 stability was enhanced in the presence of TRIM16.

\subsection{TRIM16 decreased CHIP-mediated ubiquitination degradation of RUNX2}

CHIP has been identified as a RUNX2-interacting protein. CHIP mRNA and protein levels have been shown to decrease significantly during osteogenic differentiation in МС3T3-E1 cells. To investigate the roles of CHIP in hPDLSCs, we detected CHIP protein levels during osteogenic differentiation of hPDLSCs. CHIP levels decreased significantly after osteogenic induction for 7 days (Fig. 4A). CHIP mRNA and protein levels were reduced by TRIM16 (Fig. 4B and 4C). Then a CHIP-overexpressing lentiviral vector transfected into hPDLSCs, the efficacy was determined by Western blotting (Fig. 4D). ALP and Alizarin red staining of hPDLSCs following treatment with osteogenic induction media for 7 days or 21 days showed that CHIP significantly attenuated ALP activity and delayed mineralized nodule formation, which were promoted by TRIM16 (Fig. 4E and F). To study the influence of RUNX2 on ubiquitination in the presence of CHIP and TRIM16, Myc-RUNX2 was co-transfected with HA-K48-UB, with or without Flag-CHIP and TRIM16 into HEK293T cells. CHIP significantly increased K48 linked-ubiquitination of RUNX2, which could be decreased by TRIM16, and the induction ofK48 linked-ubiquitination of RUNX2 by TRIM16 was reversed in present of CHIP (Fig. 4G). We also performed co-immunoprecipitation assays to detect interactions between CHIP and RUNX2 with or without overexpression of TRIM16, and the interactions bwteen RUNX2 and TRIM16 with or without overexpression of CHIP. The relative quantification analysis confirmed that overexpression of TRIM16 could impede the interaction between CHIP and RUNX2, overexpression of CHIP impeded the interaction between TRIM16 and RUNX2 (Fig. 4H and I), indicating that TRIM16 and CHIPmay co-regulate RUNX2 protein expression, which is critical in the TRIM16-upregulated osteogenic differentiation capacity of hPDLSCs.

\subsection{Enhanced COL1A1 expression via the p38 pathway was involved in TRIM16-associated osteogenic differentiation}

To further clarify the role of TRIM16 in late stage of osteogenic differentiation of hPDLSCs, we performed a LC-MS/MS analysis using two groups of hPDLSCs transfected with a TRIM16 or an empty lentiviral vector after incubation with osteogenic induction medium for 14 days. The principal component analysis (PCA) showed that the data from MOCK and TRIM16 cells formed two different clusters on the basis of protein expression profile (Fig. 5A).The results showed that the expression of COL1A1, COL1A1 isoform, COL1A2, COL6A1, COL6A3, and COL8A1 were significantly increased in hPDLSCs overexpressing TRIM16. COL1A1 protein levels were nearly three times greater than COL1A1 levels in the control (Fig. 5B), which was consistent with the resultsin vitro. COL1A1 is a major bone matrix protein that is primarily controlled by SP7 and RUNX2, which are well-known targets of MAPK signaling. The MAPK signaling pathway plays a vital role in bone homeostasis through phosphorylation of RUNX2 and SP7 by p38 MAPK. Activation of p38 can be blocked by CHIP. Based on previous results, we hypothesized that the activation of COL1A1 by TRIM16 during osteogenic differentiation of hPDLSCs may be influenced by $\mathrm{p} 38$ signaling. Consequently, we investigated the role of MAPK signaling in the presence of TRIM16. p38-MAPK inhibitors (SB203580) were used to suppress the p38 pathway. ALP,AR staining and the OCN protein expression showed that the osteogenic capacity of TRIM16-upregulated hPDLSCs was restricted by SB203580 treatment. Similarly, SB203580-induced inhibition of osteogenic differentiation were restored in TRIM16-overexpressing hPDLSCs (Fig. 5C-E). In addition, SB203580 suppressed COL1A1 and attenuated COL1A1 expression in TRIM16-overexpressing hPDLSCs (Fig. 5F). These observations indicate that TRIM16induced activation of COL1A1 in hPDLSCs was influenced by p38MAPK signaling. Collectively, these findings suggest that TRIM16 may facilitate osteogenic differentiation of hPDLSCs.

\section{Discussion}

Previous studies have documented the functions of TRIM16 in cancer, innate immune and other physiological and pathophysiological processes[19, 27]. To date, the role of TRIM16 in osteogenesis is largely unknown. Observations that TRIM16 expression is highly upregulated during the osteogenic media induced differentiation of hPDLSCsin vitro suggest that TRIM16 may play an important role in the osteogenic differentiation of hPDLSCs.

In this study, we reported a novel role for TRIM16 in osteogenic differentiation of hPDLSCs. TRIM16 increased the stability of RUNX2 by decreasing K48 ubiquitin-mediated proteasomal degradation, which may be attributable to the suppression of interactions between RUNX2 and CHIP. Subsequently, TRIM16 promoted the expression of COL1A1 in part through the p38MAPK/RUNX2 pathway.

RUNX2 is a key transcription factor in osteogenesis and is essential for osteogenic differentiation of suture mesenchymal cells and osteoblast progenitors. Germline deletion of RUNX2 (RUNX2-/-) or inhibition of its function in mice often results in reduced numbers of osteoblasts and marked inhibition of mineralized bone formation and chondrocyte maturation[10,11,28, 29]. Therefore, stabilization of RUNX2 is important for intramembrane and endochondral bone formation. In this study, we showed that RUNX2 protein expression was significantly enhanced in the presence of TRIM16 even though RUNX2 mRNA expression remained unchanged. These observations indicate that posttranscriptional or posttranslational modification may be involved in regulation of RUNX2. A previous study also reported a significant increase in RUNX2 protein levels with no change in mRNA expression levels during dexamethasone-

Page 5/15 
induced osteogenic differentiation[30], which may partially explain the differential effects of TRIM16 on mRNA and protein levels of RUNX2 during osteogenic differentiation of hPDLSCs.

TRIM16 belongs to the TRIM family of proteins with E3 ligase activity, however, relevant studies have shown that TRIM16 functions prominently as a regulator-receptor for the assembly of core autophagic machinery to modulate the selective autophagy process[31]. The E3 ubiquitin ligase MARCH7 is known to mediate degradation of NLRP3 in an autophagy-dependent pathway by K48-linked ubiquitination[32]. Nevertheless, TRIM16-mediated effects on RUNX2 protein expression were not altered by the autophagy inhibitor 3-MA, ruling out the possibility that TRIM16 regulates RUNX2 via an autophagy pathway. In fact, TRIM16 inhibited the reduction of RUNX2 protein relative to the control group after treated with the protein biosynthesis inhibitor CHX, and RUNX2 expression was enhanced by the proteasome inhibitor MG-132.Thus, highly dynamic post-translational modification may participate in TRIM16associated regulation of RUNX2 by the ubiquitin-proteasome system, which plays an important role in controlling the stability and activity of many proteins.

Although protein degradation may be the most recognized function of ubiquitination, the effects of ubiquitination may vary considerably depending on the topological structure of the ubiquitin chains. Seven lysine residues (K6, K11, K27, K29, K33, K48, and K63) and the N-terminal methionine residue (M1) are capable of acting as receptors for conjugation, producing polyubiquitin chains, which produce different ubiquitination results in different substrates[33]. The K48-linked chains normally serve as signals for proteasomal degradation. In contrast, K63-linked chains may also mediate the activation and re-localization of certain protein kinases to function in other proteasome-independent cellular processes[34, 35]. For example, the E3 ligase RSP5 positively regulates the osteogenic capacity of MSCs by inducing K63-linked polyubiquitination and activating Akt[36]. WWP2 facilitated transactivation of RUNX2 by affecting the mono-ubiquitination during osteogenic differentiation[15].

In previous studies, TRIM16 has been reported to decrease K48-linked poly-ubiquitination and increase K63-linked poly-ubiquitination of NRF2. TRIM16 facilitated displacement and degradation of KEAP1, which is a negative regulator of NRF2 stability[37]. We investigated the effects of TRIM16 on the ubiquitination of RUNX2 and inferred that there was a negative regulator of RUNX2. As expected, TRIM16 decreased K48-linked poly-ubiquitination of RUNX2, which is a known principal delivery signal for proteasomal degradation. Several ubiquitin E3 ligases, including SMURF1, CHIP and WWP1 have been shown to interact directly with RUNX2 and induce ubiquitin-dependent degradation of RUNX2 during commitment to the osteoblast lineage[38, 39]. However, SMURF1 remains unchanged during osteogenic differentiation, and elevated WWP1 in late stages of osteoblast differentiation appears to enhance RUNX2 degradation. CHIPprotein levels decrease steadily accompanied by an increase in RUNX2 protein levels and restrict osteogenic differentiation[18].Diminished levels of RUNX2 and increased K48-linked poly-ubiquitination were observed in hPDLSCs overexpressing CHIP. Of note, both TRIM16 and CHIP bind to RUNX2, andTRIM16 impeded the interaction between CHIP and RUNX2, which might be a critical component of their interrelationship. Accordingly, TRIM16 promoted RUNX2 stability by decreasing CHIP-mediated K48-linked poly-ubiquitination of RUNX2.

RUNX2 is required for the induction of several major bone matrix protein genes, including COL1A1, BGLAP2 and FN1 along with other osteogenic transcription factors such as Sp7 and DIx5[40-42]. In our study, TRIM16 significantly increased the expression of COL1A1, COL1A1 isoform, COL1A2, COL6A1, COL6A3 and COL8A1. The LC-MS/MS analysis, which focused on changes in collagen production due to TRIM16, provided a better understanding of the diverse mechanisms of TRIM16 osteogenesis. COL1A1 and COL1A2 are responsible for encoding collagen I, a heterotrimeric protein comprised of two a1 (I) chains and one a2(I) chain, which is the most abundant protein of the bone extracellular matrix, accounting for $90 \%$ of the matrix protein content[43]. It has been well established that Sp7 and RUNX2 are involved in COL1A1 expression in vitro, which is augmented through phosphorylation by p38 and ERK[41, 44]. Activation of p38MAPK has been reported to be blocked by CHIP $[45,46]$. In this study, we further investigated the role of p38 in modulating COL1A1 in the presence of TRIM16. We confirmed that P38MAPK is essential in TRIM16-induced activation of COL1A1 in hPDLSCs. Increased COL1A1 expression in the presence of TRIM16 further demonstrated a positive role of TRIM16 in osteogenic differentiation of hPDLSCs. Recently, mechanisms of TRIM16 and coregulation in osteogenic differentiation of hBMSCs have been reported, which is consistent with our results[47].

In contrast to previous studies which showed that ubiquitin E3 ligase exerts inhibitory effects on osteogenic differentiation in vitro, this study discovered that TRIM16 promotes osteogenic differentiation of hPDLSCs by stabilizing RUNX2. There is a decrease in CHIP expression in present of TRIM16 to slow the degradation of its substrate RUNX2, and also a compete between TRIM16 and CHIP for binding directly to RUNX2. The similar mode of regulation was observed in another study where LMCD1 protected the RUNX2 protein from SMURF1-mediated ubiquitination degradation, thereby regulating BMP signaling[48]. Recently, the CK2/HAUSP pathway was reported to be a key regulator of RUNX2 stability by a de-ubiquitination pathway[49]; Thus,a protein known as a de-ubiquitinase may exist, which can be recruited by TRIM16 to cleave K48-linked poly-ubiquitination chains from RUNX2, however, few studies have reported the de-ubiquitination of RUNX2, which need to be further explored.

\section{Conclusion}

In summary, our study provided the positive evidence that TRIM16 plays a critical role in regulating osteogenic differentiation of hPDLSCs by modulating protein levels of RUNX2 through ubiquitination and protein degradation. Additionally, we found that TRIM16 is important for promoting the expression of COL1A1 via the p38MAPK/RUNX2 pathway. Therapeutic targeting of TRIM16 may be a promising strategy for bone regeneration in periodontitis.

\section{Abbreviations}

hPDLSCs:human periodontal ligament stem cells

MSCs :Mesenchymal stem cells

ALP :Alkaline phosphatase 
ARS :alizarin red staining

ELISA :enzyme-linked immunosorbent assays

TRIM :the tripartite motif

shRNAs:specific short-hairpin RNAs

CHIP :carboxy terminus of HSP70 interacting protein

EMT :epithelial-to-mesenchymal transition

FBS :fetal bovine serum

ORF :open reading frame

PVDF :polyvinylidene fluoride

OCN :osteocalcin

FASP :filter aided sample preparation

HA :hemagglutinin

$\mathrm{CHX}$ :cycloheximide

\section{Declarations}

\section{Availability of data and materials}

The datasets used and/or analyzed during the current study are available from the

corresponding author on reasonable request.

\section{Acknowledgments}

Not applicable.

\section{Funding}

This work was supported by The National Natural Science Foundation of China (grant number 81571010), Key Research and Development project of Shandong Province (grant number 2018GSF118199), and Graduate education outstanding achievement training program of Shandong University(grant number ZY20190004)

\section{Ethics approval and consent to participate}

All experiments have been authorized by the Medical Ethics Committee of the School of Stomatology, Shandong University. Informed consent was collected from all participants involved in this study, and all procedures were performed in accordance with the guidelines.

\section{Consent for publication}

Not applicable.

\section{Authors' contributions}

DL and $Y Z$ conceived the project design and conception. $Y Z$ andQZ were responsible for the experimental design and application including data acquisition and analysis. YZ and HL wrote and revised the manuscript. XX and SC standardized the figures. DL provided valuable suggestions for the manuscript. All authors gave final approval of the manuscript and agreed to be accountable for all aspects of the work.

\section{Competing interests}

The authors declare that they have no competing interests.

\section{Reference}

1. Kinane DF,Stathopoulou PG,Papapanou PN. Periodontal diseases. Nat Rev Dis Primers 2017;3:17038.

2. Sculean A, Nikolidakis D, Nikou G, Ivanovic A, Chapple IL, Stavropoulos A. Biomaterials for promoting periodontal regeneration in human intrabony defects: a systematic review. Periodontol 2000 2015;68(1):182-216.

3. Sedgley CM, Botero TM. Dental stem cells and their sources. Dent Clin North Am 2012;56(3):549-561. 
4. Bojic S, Volarevic V, Ljujic B, Stojkovic M. Dental stem cells-characteristics and potential. Histol Histopathol 2014;29(6):699-706.

5. Park JY, Jeon SH, Choung PH. Efficacy of periodontal stem cell transplantation in the treatment of advanced periodontitis. Cell Transplant 2011;20(2):271-285.

6. Bartold PM, Gronthos S, Ivanovski S, Fisher A, Hutmacher DW. Tissue engineered periodontal products. J Periodontal Res 2016;51(1):1-15.

7. Moon JS, Kim MJ, Ko HM, Kim YJ, Jung JY, Kim JH, Kim SH, Kim MS. The role of Hedgehog signaling in cementoblast differentiation. Arch Oral Biol 2018;90:100-107.

8. Yu F, Wu F, Li F, Liao X, Wang Y, Ye L, et al. Wnt7b-induced Sox11 functions enhance self-renewal and osteogenic commitment of bone marrow mesenchymal stem cells. Stem Cells 2020.

9. Zhang Y, Yang C, Ge S, Wang L, Zhang J, Yang P. EphB4/ TNFR2/ERK/MAPK signaling pathway comprises a signaling axis to mediate the positive effect of TNF-alpha on osteogenic differentiation. BMC Mol Cell Biol 2020;21(1):29.

10. Komori T, Yagi H, Nomura S, Yamaguchi A, Sasaki K, Deguchi K, et al. Targeted disruption of Cbfa1 results in a complete lack of bone formation owing to maturational arrest of osteoblasts. Cell 1997;89(5):755-764.

11. Qin X, Jiang Q, Miyazaki T, Komori T. Runx2 regulates cranial suture closure by inducing hedgehog, Fgf, Wnt and Pthlh signaling pathway gene expressions in suture mesenchymal cells. Hum Mol Genet 2019;28(6):896-911.

12. Gomathi K, Akshaya N, Srinaath N, Moorthi A, Selvamurugan N. Regulation of Runx2 by post-translational modifications in osteoblast differentiation. Life Sci 2020;245:117389.

13. Kim HJ, Kim WJ, Ryoo HM. Post-Translational Regulations of Transcriptional Activity of RUNX2. Mol Cells 2020;43(2):160-167.

14. Komori T. Molecular Mechanism of Runx2-Dependent Bone Development. Mol Cells 2020;43(2):168-175.

15. Zhu W, He X, Hua Y, Li Q, Wang J, Gan X. The E3 ubiquitin ligase WWP2 facilitates RUNX2 protein transactivation in a mono-ubiquitination manner during osteogenic differentiation. J Biol Chem 2017;292(27):11178-11188.

16. Kumar Y, Kapoor I, Khan K, Thacker G, Khan MP, Shukla N, et al. E3 Ubiquitin Ligase Fbw7 Negatively Regulates Osteoblast Differentiation by Targeting Runx2 for Degradation. J Biol Chem 2015;290(52):30975-30987.

17. Liu J, Li X, Zhang H, Gu R, Wang Z, Gao Z, Xing L. Ubiquitin E3 ligase Itch negatively regulates osteoblast function by promoting proteasome degradation of osteogenic proteins. Bone Joint Res 2017;6(3):154-161.

18. Li X, Huang M, Zheng H, Wang Y, Ren F, Shang Y, et al. CHIP promotes Runx2 degradation and negatively regulates osteoblast differentiation. J Cell Biol 2008;181(6):959-972.

19. Hatakeyama S. TRIM Family Proteins: Roles in Autophagy, Immunity, and Carcinogenesis. Trends Biochem Sci 2017;42(4):297-311.

20. Bell JL, Malyukova A, Holien JK, KoachJ, Parker MW, Cheung BB, et al. TRIM16 acts as an E3 ubiquitin ligase and can heterodimerize with other TRIM family members. PLoS One 2012;7(5):e37470.

21. Bell JL, Malyukova A, Kavallaris M, Marshall GM, Cheung BB. TRIM16 inhibits neuroblastoma cell proliferation through cell cycle regulation and dynamic nuclear localization. Cell Cycle 2013;12(6):889-898.

22. Huo X, Li S, Shi T, Suo A, Ruan Z, Yao Y. Tripartite motif 16 inhibits epithelial-mesenchymal transition and metastasis by down-regulating sonic hedgehog pathway in non-small cell lung cancer cells. Biochem Biophys Res Commun 2015;460(4):1021-1028.

23. Qi L, Lu Z, Sun YH, Song HT, Xu WK. TRIM16 suppresses the progression of prostate tumors by inhibiting the Snail signaling pathway. Int J Mol Med 2016;38(6):1734-1742.

24. Kumar S, Chauhan S, Jain A, Ponpuak M, Choi SW, Mudd M, et al. Galectins and TRIMs directly interact and orchestrate autophagic response to endomembrane damage. Autophagy 2017;13(6):1086-1087.

25. Jena KK, Mehto S, Kolapalli SP, Nath P, Sahu R, Chauhan NR, et al. TRIM16 governs the biogenesis and disposal of stress-induced protein aggregates to evade cytotoxicity: implication for neurodegeneration and cancer. Autophagy 2019;15(5):924-926.

26. Aral K, Berdeli E, Cooper PR, Milward MR, Kapila Y, Berdeli A, et al. Differential expression of inflammasome regulatory transcripts in periodontal disease. J Periodontol 2020;91(5):606-616.

27. Sutton SK, Cheung BB, Massudi H, Tan O, Koach J, Marshall GM, et al. Heterozygous loss of keratinocyte TRIM16 expression increases melanocytic cell lesions and lymph node metastasis. J Cancer Res Clin Oncol 2019;145(9):2241-2250.

28. Maruyama Z, Yoshida CA, Furuichi T, Amizuka N, Ito M, Fukuyama R, et al. Runx2 determines bone maturity and turnover rate in postnatal bone development and is involved in bone loss in estrogen deficiency. Dev Dyn 2007;236(7):1876-1890.

29. Otto F, Thornell AP, Crompton T, Denzel A, Gilmour KC, Rosewell IR, et al. Cbfa1, a candidate gene for cleidocranial dysplasia syndrome, is essential for osteoblast differentiation and bone development. Cell 1997;89(5):765-771.

30. Prince M, Banerjee C, Javed A, Green J, Lian JB, Komm BS, et al. Expression and regulation of Runx2/Cbfa1 and osteoblast phenotypic markers during the growth and differentiation of human osteoblasts. J Cell Biochem 2001;80(3):424-440.

31. Kimura T, Jia J, Claude-Taupin A, Kumar S, Choi SW, GuY, et al. Cellular and molecular mechanism for secretory autophagy. Autophagy 2017;13(6):10841085.

32. Yan Y, Jiang W, Liu L, Wang X, Ding C, Tian Z, Zhou R. Dopamine controls systemic inflammation through inhibition of NLRP3 inflammasome. Cell 2015;160(1-2):62-73.

33. Swatek KN, Komander D. Ubiquitin modifications. Cell Res 2016;26(4):399-422.

34. van Huizen M, Kikkert M. The Role of Atypical Ubiquitin Chains in the Regulation of the Antiviral Innate Immune Response. Front Cell Dev Biol $2019 ; 7: 392$. 
35. Oualid FE, Hameed DS, Atmioui DE, Hilkmann H, Ovaa H. Synthesis of atypical diubiquitin chains. Methods Mol Biol 2012;832:597-609.

36. Liang C, Liang G, Zheng X, Huang Y, Huang S, Yin D. RSP5 Positively Regulates the Osteogenic Differentiation of Mesenchymal Stem Cells by Activating the K63-Linked Ubiquitination of Akt. Stem Cells Int 2020;2020:7073805.

37. Jena KK, Kolapalli SP, Mehto S, Nath P, Das B, Sahoo PK, et al. TRIM16 controls assembly and degradation of protein aggregates by modulating the p62NRF2 axis and autophagy. EMBO J 2018;37(18).

38. Shimazu J, Wei J, Karsenty G. Smurf1 Inhibits Osteoblast Differentiation, Bone Formation, and Glucose Homeostasis through Serine 148. Cell Rep 2016;15(1):27-35.

39. Shu L, Zhang H, Boyce BF, Xing L. Ubiquitin E3 ligase Wwp1 negatively regulates osteoblast function by inhibiting osteoblast differentiation and migration. J Bone Miner Res 2013;28(9):1925-1935.

40. Ducy P, Zhang R, Geoffroy V, Ridall AL, Karsenty G. Osf2/Cbfa1: a transcriptional activator of osteoblast differentiation. Cell 1997;89(5):747-754.

41. Komori T. Regulation of Proliferation, Differentiation and Functions of Osteoblasts by Runx2. Int J Mol Sci 2019;20(7).

42. Komori T. Roles of Runx2 in Skeletal Development. Adv Exp Med Biol 2017;962:83-93.

43. Kern B, Shen J, Starbuck M, Karsenty G. Cbfa1 contributes to the osteoblast-specific expression of type I collagen genes. J Biol Chem 2001;276(10):71017107.

44. Artigas N, Urena C, Rodriguez-Carballo E, Rosa JL, Ventura F. Mitogen-activated protein kinase (MAPK)-regulated interactions between Osterix and Runx2 are critical for the transcriptional osteogenic program. J Biol Chem 2014;289(39):27105-27117.

45. Yang K, Zhang TP, Tian C, Jia LX, Du J, Li HH. Carboxyl terminus of heat shock protein 70-interacting protein inhibits angiotensin II-induced cardiac remodeling. Am J Hypertens 2012;25(9):994-1001.

46. Xiong W, Liu S, Cai W, Wen J, FuY, Peng J, Zheng Z. The carboxyl terminus of heat shock protein 70-interacting protein (CHIP) participates in high glucoseinduced cardiac injury. Free Radic Biol Med 2017;106:339-344.

47. Chen WT, Zhang F, Zhao XQ, Yu B, Wang BW. Galectin-3 and TRIM16 coregulate osteogenic differentiation of human bone marrow-derived mesenchymal stem cells at least partly via enhancing autophagy. Bone 2020;131:115059.

48. Zhu B, Xue F, Zhang C, Li G. LMCD1 promotes osteogenic differentiation of human bone marrow stem cells by regulating BMP signaling. Cell Death Dis 2019;10(9):647.

49. Kim JM, Yang YS, Park KH, Ge X, Xu R, Li N, et al. A RUNX2 stabilization pathway mediates physiologic and pathologic bone formation. Nat Commun 2020;11(1):2289.

\section{Tables}

Table1

Primers used in plasmid construction

\begin{tabular}{|lll}
\hline Genes & Forward $\left(5^{\prime} \rightarrow 3^{\prime}\right)$ & Reverse $\left(5^{\prime} \rightarrow 3^{\prime}\right)$ \\
\hline TRIM16 & GACTCTCGAGGCCACCATGGC & GACTGAATTCCTAGGGAGCAGTCCCCACCAAG \\
& GAGTTGGATCTAA & AATTCAAAAAGCAGTGAAGTCCTGTCTAACCCTCGAGG \\
shTRI6 & CCGGGCAGTGAAGTCCTGTCTAACCCTCGAGGGTTAGACAGGACTTCACTGCTTTTTG & AGTCGAATTCGCCACCATGGATTACAAGGATGACGACG \\
& AGTCGGATCCTCAGTAGTCCTCCACCCAGCCATTC &
\end{tabular}

CHIP

Table2

Primers used in RT-qPCR

\begin{tabular}{|lll|}
\hline Genes & Forward $\left(5^{\prime} \rightarrow 3^{\prime}\right)$ & Reverse $\left(5^{\prime} \rightarrow 3^{\prime}\right)$ \\
\hline RUNX2 & CACTGGCGCTGCAACAAGA & CATTCCGGAGCTCAGCAGAATA \\
SP7 & ATGCCAACACAGTGTTGTCTGG & TACTCCTGCTTGCTGATCCACAT \\
COL1A1 & GGCCTAAGGGTGACAGAGGT & AGTCAGACCACGGACGCCAT \\
\hline
\end{tabular}




\section{Figures}

Fig.1

A
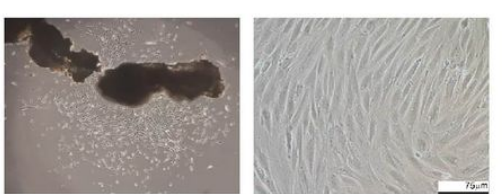

B

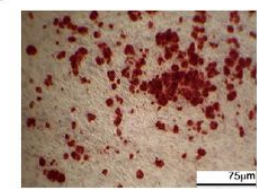

D

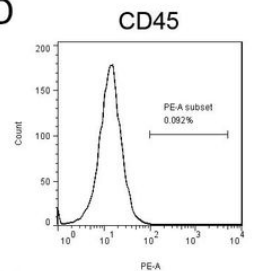

E

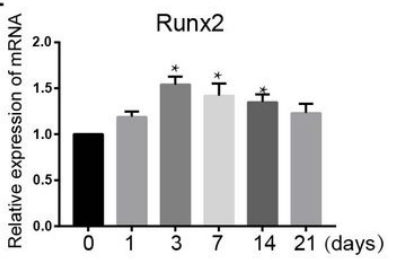

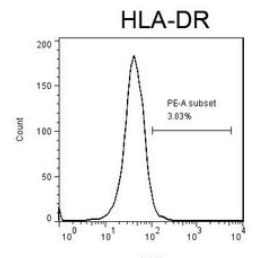

PE,

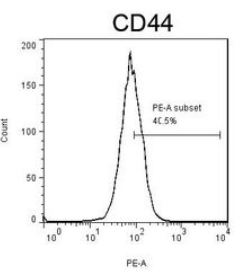

$P E A$

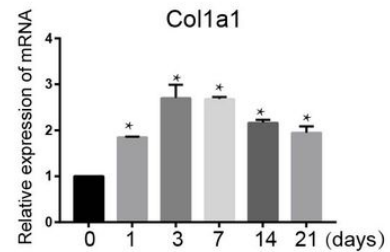

C
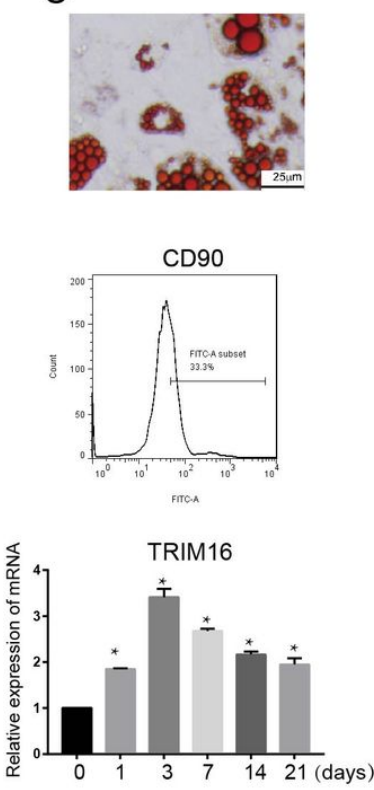
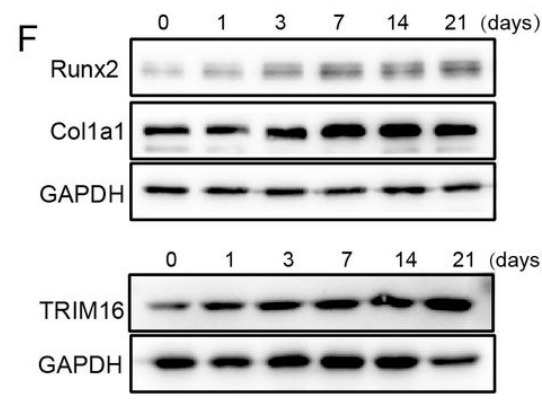

G 0

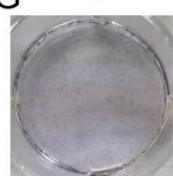

0

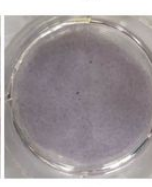

7

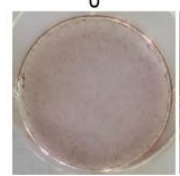

7

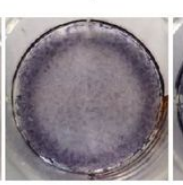

14

(days)

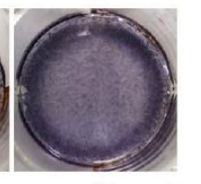

14

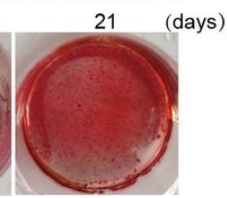

\section{Figure 1}

TRIM16 expression is upregulated during osteogenic differentiation of hPDLSCs. (A) hPDLSCs were derived from the periodontal ligament and cultured from passage 3 to passage 7. (B) Alizarin Red-positive mineralized matrix and (C) Oil Red O-positive lipid droplets were shown to possess multi-differentiation capacity. (D) MSC-specific surface markers were detected by flow cytometry. (E) mRNA, (F) protein expression of TRIM16 and (G) ALP staining and Alizarin red staining during osteogenic differentiation of hPDLSCs was examined by Western blotting. * $\mathrm{P}<0.05$ by Student's t-test. 
Fig.2

A
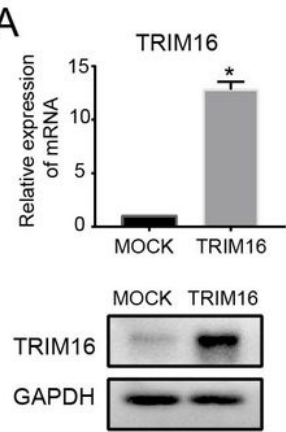

E

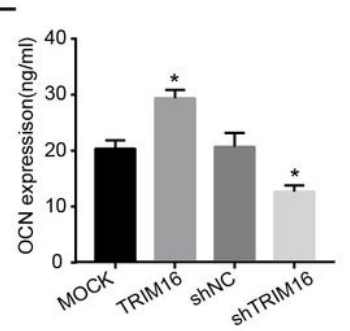

G

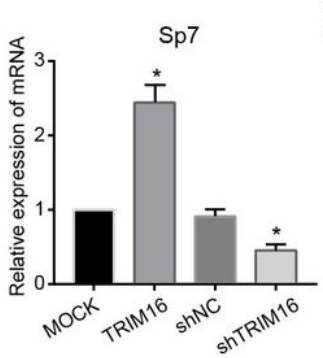

B
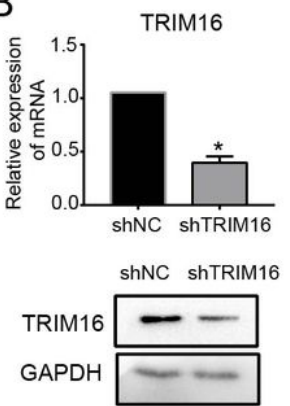

C

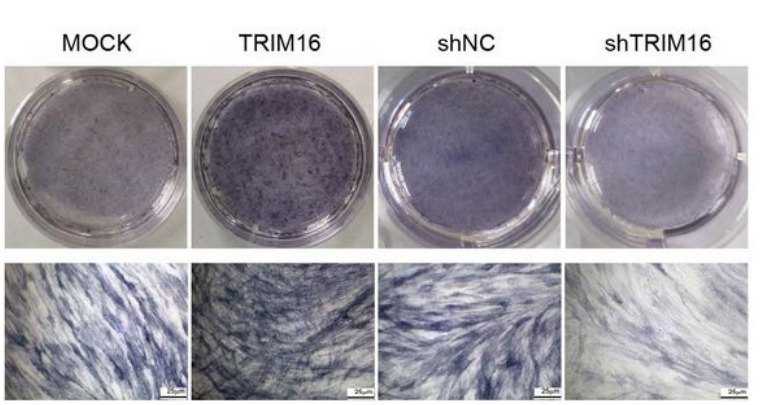

D

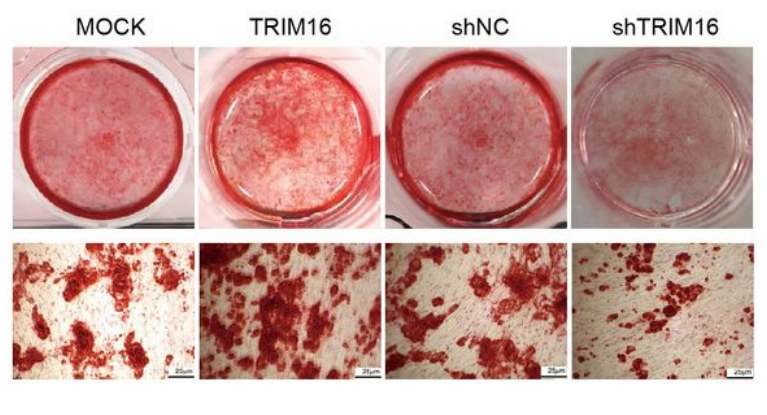

\section{Figure 2}

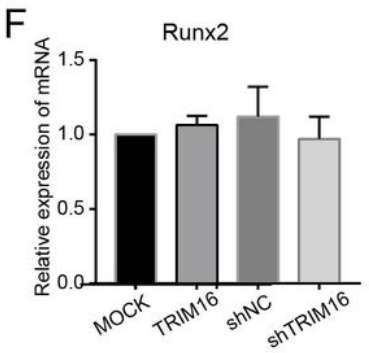

\section{I}
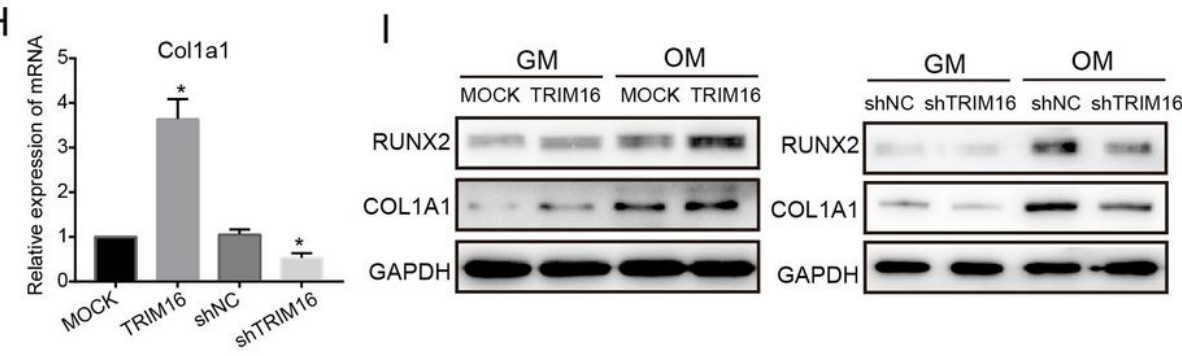

TRIM16 promoted osteogenic differentiation of hPDLSCs. (A) The efficacy of a TRIM16-overexpressing lentiviral vector and (B) a TRIM16 specific shRNA were measured by RT-qPCR and Western blotting. (C) ALP staining and (D) Alizarin red staining were performed after treating hPDLSCs with osteogenic induction media for 7 days or 14 days. (E) OCN levels were measured by an ELISA assay. The expression of osteogenic markers (F) RUNX2, (G) Sp7 and (H) COL1A1 was assessed by RT-qPCR. (I) The protein expression of osteogenic markers RUNX2, COL1A1 was assessed by Western blotting. *P<0.05 by Student's t-test. 
Fig.3

A

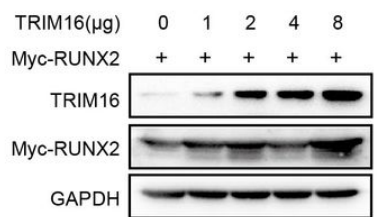

C

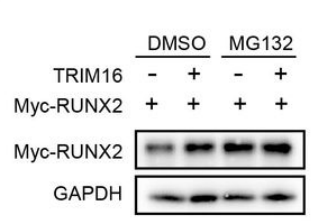

D

TRIM16 - $-\quad+++$

Myc-RUNX2 ++++++

3-MA(mM) $\quad \begin{array}{llllll}0 & 5 & 10 & 0 & 5 & 10\end{array}$

Myc-RUNX2 $=$

GAPDH $-\infty---$

B
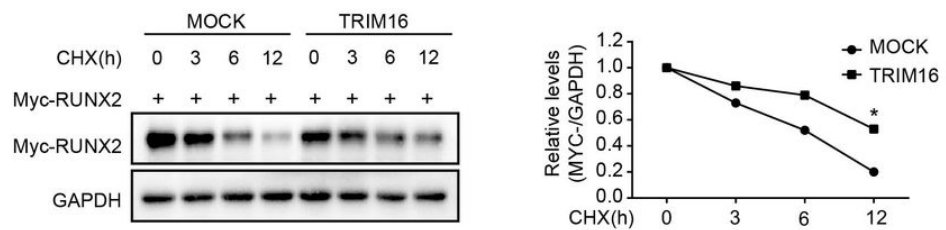

E

Myc-RUNX2 + +

$\mathrm{HA}-\mathrm{Ub}++$

TRIM16 - +

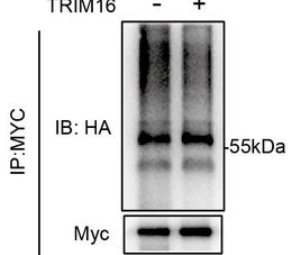

F
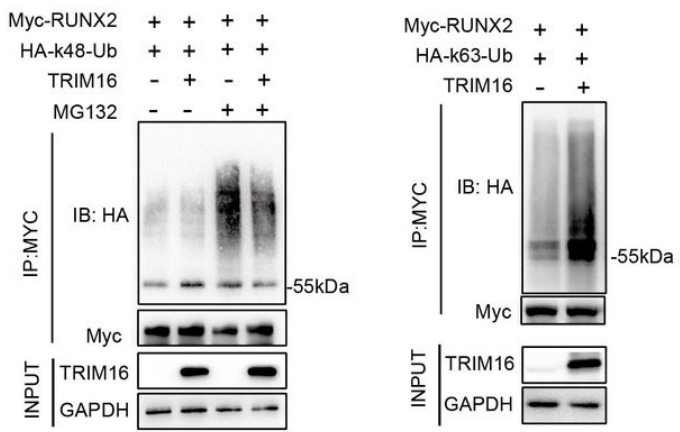

G

\begin{tabular}{l|l} 
与 & TRIM16 \\
$\underline{\underline{n}}$ & GAPDH
\end{tabular}

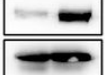

$\underline{\underline{n}}{ }_{\text {GAPDH }}-$

$\mathrm{H}$
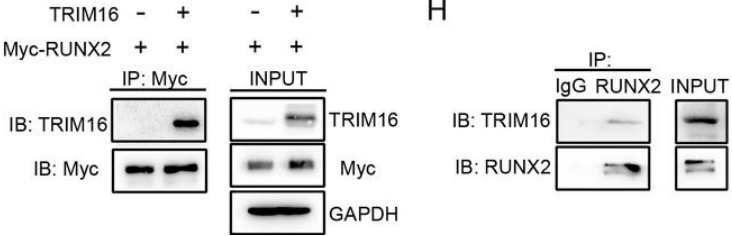

\section{Figure 3}

TRIM16 reduced the ubiquitination degradation of RUNX2. (A) Immunoblot analysis of extracts from HEK293T cells transfected with Myc-RUNX2 and different doses of a TRIM16 overexpression plasmid. (B) Immunoblot analysis of extracts from HEK293T cells transfected with Myc-RUNX2 and a TRIM16 overexpression plasmid or control vector treated with cycloheximide (CHX) for various times. The expression of RUNX2 and TRIM16 were quantitated by measuring band intensities using 'ImageJ' software. Values were normalized to GADPH. (C) Immunoblot analysis of extracts from HEK293T cells transfected with Myc-RUNX2 and a TRIM16 overexpression plasmid then treated with MG132 (10 mM) for $4 \mathrm{~h}$. (D) Immunoblot analysis of extracts from HEK293T cells transfected with Myc-RUNX2 and a TRIM16 overexpression plasmid or control vector treated with the indicated concentration of 3-MA for $4 \mathrm{~h}$. (E, F) Immunoblot analysis of lysates from HEK293T cells transfected with Myc-RUNX2 and a TRIM16 overexpression plasmid or control vector, HA-tagged ubiquitin (HA-Ub) or HA-tagged K48-linked polyubiquitination (HA-K48-Ub), HA-tagged K63-linked polyubiquitination (HA-K63-Ub), followed by IP with anti-Myc, and probed with anti-HA. (G) Coimmunoprecipitation was performed to detect the interaction of TRIM16 and RUNX2, followed by IP with anti-Myc, and probed with anti-TRIM16 in HEK293T cells and (H) in hPDLSCs. *P<0.05 by Student's t-test. 
Fig.4
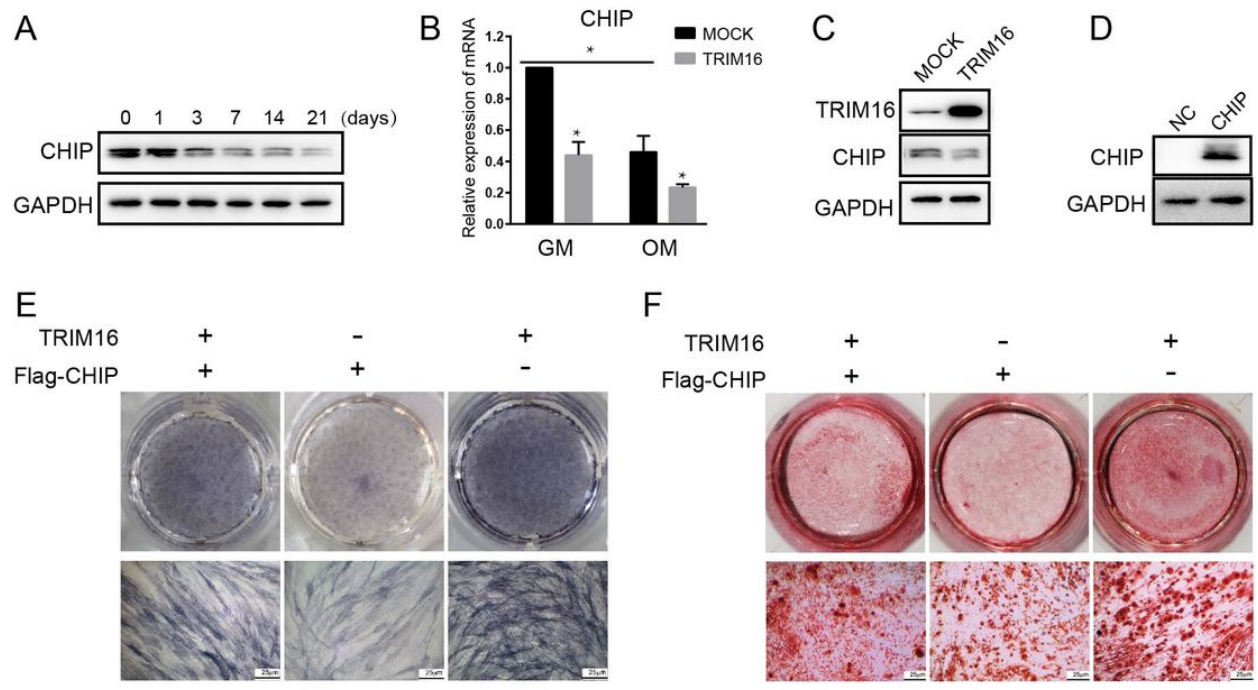

F
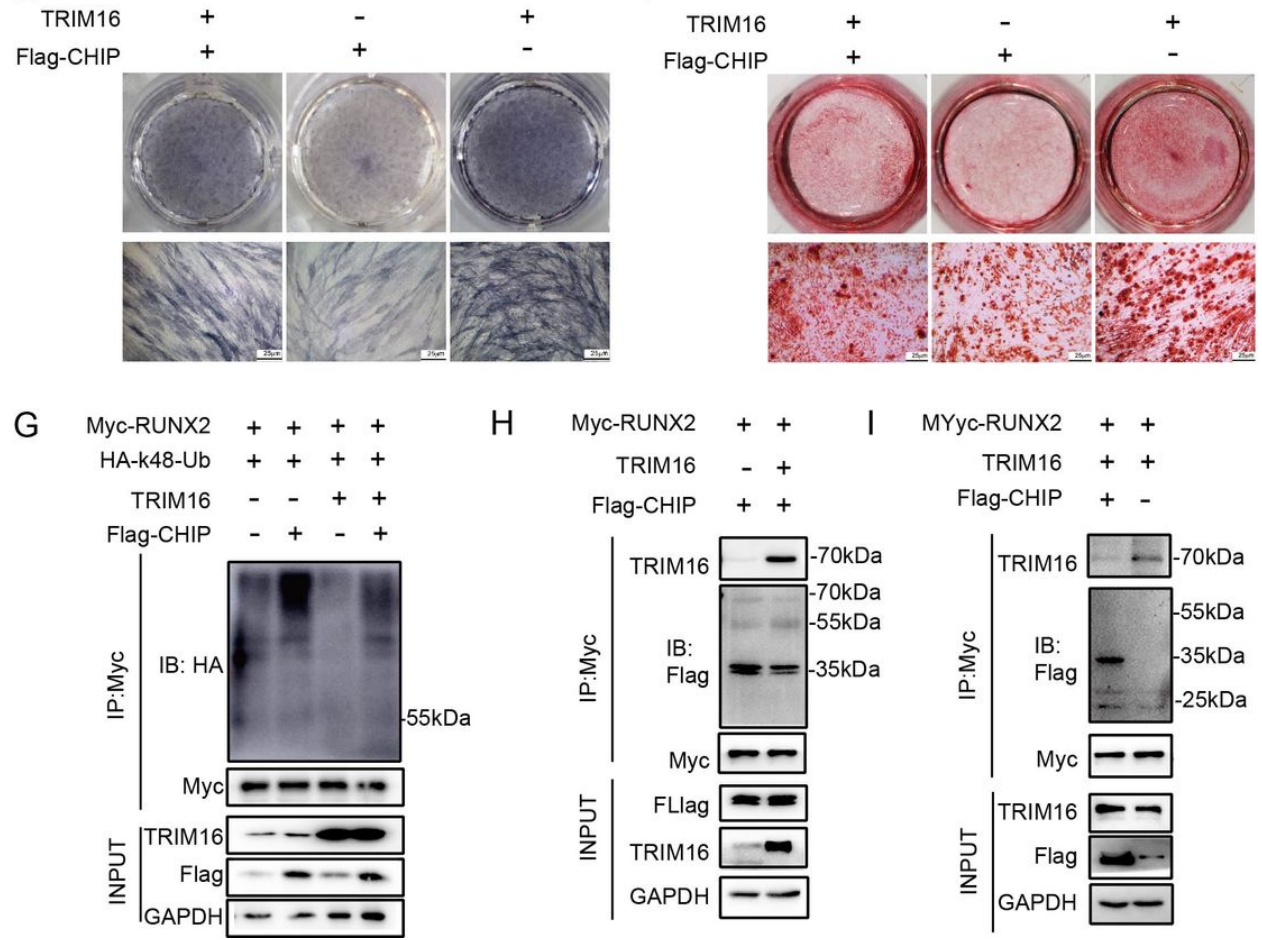

\section{Figure 4}

TRIM16 decreased CHIP-mediated ubiquitination degradation of RUNX2 (A) Expression of CHIP during osteogenic differentiation of hPDLSCs was examined by Western blotting. (B) mRNA and (C)protein levels of CHIP with or without overexpression of TRIM16. (D)The efficacy of a CHIP-overexpressing lentiviral vector was measured by Western blotting. (E) ALP staining and (F) Alizarin red staining were performed after treating hPDLSCs with osteogenic induction media for 7 days or 14 days. (G) Immunoblot analysis of lysates from HEK293T cells transfected with Myc-RUNX2, HA-tagged K48-linked polyubiquitination (HA-K48-Ub) with or without a TRIM16 overexpression plasmid or Flag-CHIP, followed by IP with anti-Myc, and probed with anti-HA. (H) Coimmunoprecipitation was performed to detect the interaction of CHIP and RUNX2. HEK293T cells transfected with Myc-RUNX2, Flag-CHIP, a TRIM16 overexpression plasmid or control vector, followed by IP with anti-Myc, and probed with anti-Flag. (I) Coimmunoprecipitation was performed to detect the interaction of TRIM16 and RUNX2. HEK293T cells transfected with Myc-RUNX2, a TRIM16 overexpression plasmid with or without Flag-CHIP, followed by IP with anti-Myc, and probed with anti-TRIM16. ${ }^{*}<0.05$ by Student's t-test. 
Fig.5

A

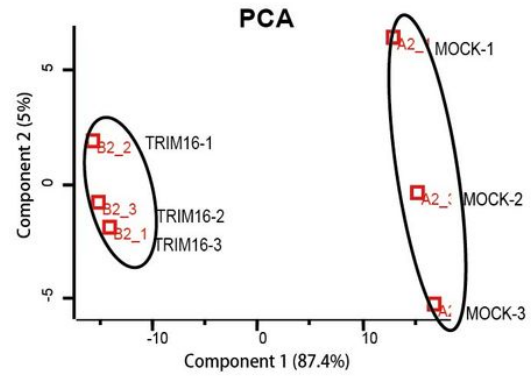

D TRIM16
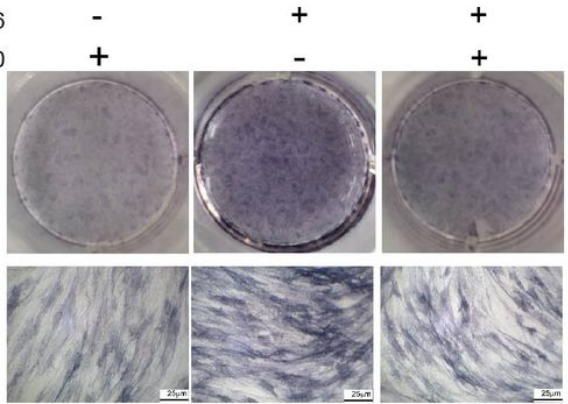

E

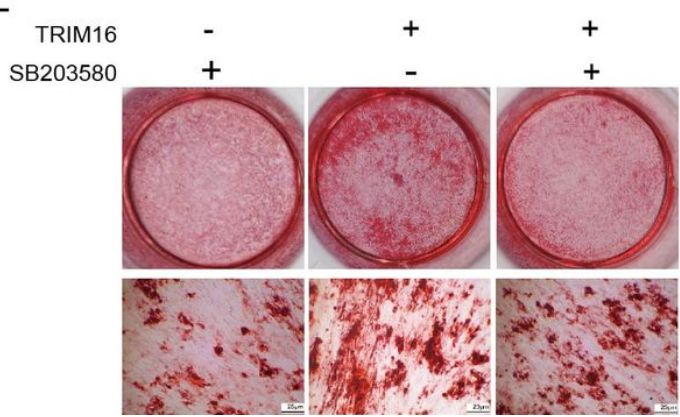

B

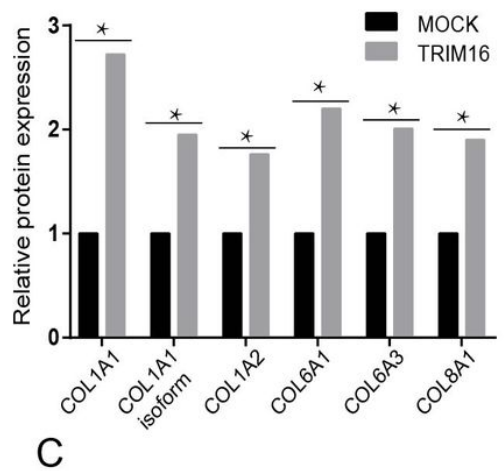

C

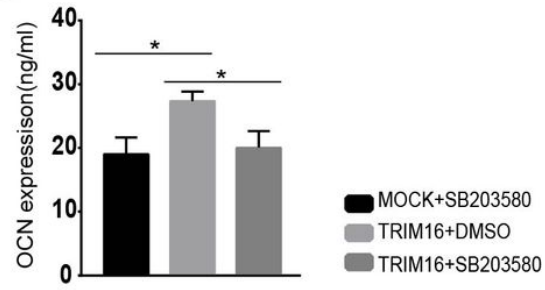

$\mathrm{F}$

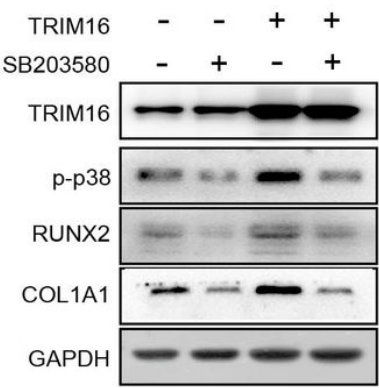

Figure 5

Enhanced COL1A1 expression via the p38 pathway was involved in TRIM16 associated osteogenic differentiation. (A) The principal component analysis (PCA) showed two different clusters on the basis of protein expression profile.(B) The relative expression of collagen subfamily proteins according to LCMS/MS analysis. (C) OCN levels were measured by an ELISA assay. (D) ALP staining and (E) Alizarin red staining were performed in the presence of TRIM16, with or without SB203580 treatment. (F) The expression of p38, RUNX2 and COL1A1 was measured by Western blotting in the presence of TRIM16 or a control vector, with or without SB203580 treatment. ${ }^{*} \mathrm{P}<0.05$ by Student's $t$-test. 
Fig. 6

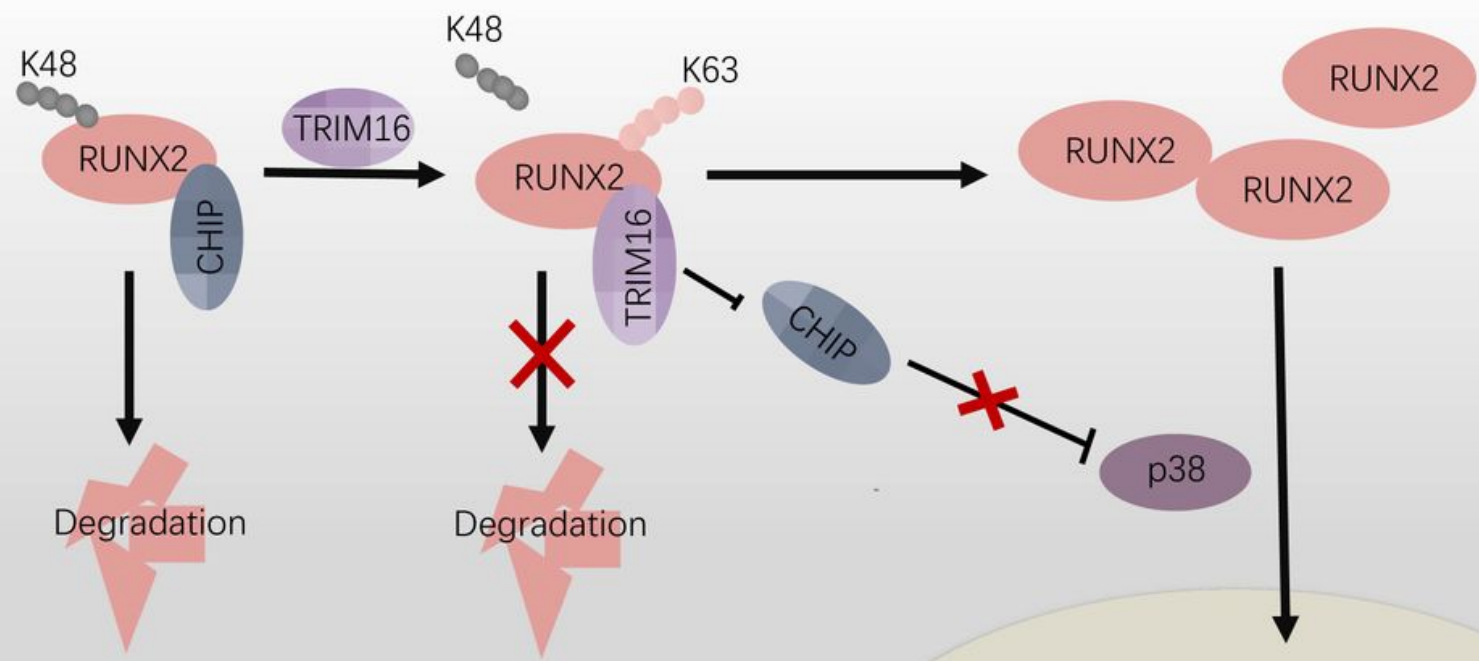

SP7

COL1A1

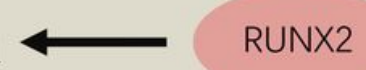

racacacacal

\section{Figure 6}

A model illustrating that TRIM16 promotes osteogenic differentiation via stability of RUNX2. Overexpression of TRIM16 increases the stability of RUNX2 by decreasing CHIP-mediated K48-linked polyubiquitination and further promotes osteogenic differentiation of hPDLSCs in part through activation of p38. 